\title{
PD-LI and miR-34a are Prognostic Factors for Primary Gastric Diffuse Large B-Cell Lymphoma Patients Treated with R-CHOP
}

This article was published in the following Dove Press journal: Cancer Management and Research

\author{
Jinfeng Wang,' Song Shang,' \\ Junjun Li,' Hongyu Deng,' \\ Linda Ouyang, ' Hailong Xie, ${ }^{2}$ \\ Haizhen Zhu, ${ }^{3}$ Yajun Li, \\ Chaohui Zuo (iD)' \\ 'Department of Gastroduodenal and \\ Pancreatic Surgery, Translation Medicine \\ Research Center of Liver Cancer, \\ Laboratory of Digestive Oncology, \\ Affiliated Cancer Hospital of Xiangya \\ Medical School \& Hunan Cancer \\ Hospital, Central South University, \\ Changsha, People's Republic of China; \\ ${ }^{2}$ Graduates School, Cancer Research \\ Institute, University of South China, \\ Hengyang, People's Republic of China; \\ ${ }^{3}$ Institute of Pathogen Biology and \\ Immunology of College of Biology, State \\ Key Laboratory of Chemo/Biosensing and \\ Chemometrics, Hunan University, \\ Changsha, People's Republic of China
}

Correspondence: Chaohui Zuo Department of Gastroduodenal and Pancreatic Surgery, Laboratory of Digestive Oncology, Affiliated Tumor Hospital of Xiangya Medical School \& Hunan Cancer Hospital, Central South University, Hunan University- Hunan Province Tumor Hospital Joint Center of Liver Cancer Translational Medicine, Changsha, People's Republic of China Tel +8673 I89762 I40

Email zuochaohui@vip.sina.com

Yajun Li

Department of Gastroduodenal and Pancreatic Surgery, Laboratory of Digestive Oncology, Affiliated Tumor Hospital of Xiangya Medical School \& Hunan Cancer Hospital, Central South University, Changsha, People's Republic of China

Tel +8673l89762140

Email liyajun2391@I63.com
Introduction: Primary gastric diffuse large B-cell lymphoma (GDLBCL) is a heterogeneous disease in clinicopathological features and prognosis. Programmed death ligand-1 (PD-L1) and microRNA-34a (miR-34a) play crucial roles in GDLBCL progress. The purpose of this research is to explore the clinical significance of PD-L1 and miR-34a expression in GDLBCL.

Patients and Methods: The expressions of PD-L1 and miR-34a were examined by IHC and qRT-PCR in 109 patients who were diagnosed with GDLBCL and were treated with rituximab plus cyclophosphamide, doxorubicin, prednisone vincristine and prednisone chemotherapy (R-CHOP) from January 2010 to December 2018.

Results: PD-L1 level was significantly higher in tumor tissues than adjacent non-tumor tissues $(60.5 \%, P<0.001)$, while the miR-34a level was just reversed $(50.5 \%, P<0.001)$, which was negatively correlated $(r=-0.524, P<0.001)$. Notably, PD-L1-positive and miR34a-negative expressions were significantly correlated with the advanced Lugano stage of IIE-IV stage $(P<0.001$ and $P<0.01)$, elevated serumal LDH levels $(P<0.001$ and $P<0.05)$, B symptoms present $(P<0.001$ and $P<0.001)$, non-GCB subtype $(P<0.001$ and $P<0.001)$ and negative Bcl-2 expression $(P<0.05$ and $P<0.001)$. PD-L1 high and miR-34a low expression groups had more patients with IPI scores of 2 or greater $(P<0.001$ and $P<0.05)$ and poor R-IPI $(P<0.01$ and $P<0.01)$. The complete response rate was upregulated in patients with negative PD-L1 and positive miR-34a expression after R-CHOP treatment.

Discussion: PD-L1 expression and miR-34a expression were significantly associated with clinicopathological characteristics and survival prognosis; they may serve as novel prognostic markers in GDLBCL patients who were treated with R-CHOP. Immunotherapies targeting PD-L1 and miR-34a pathway may have therapeutic potential in GDLBCL.

Keywords: GDLBCL, R-CHOP, PD-L1, miR-34a, tumor immunotherapy

\section{Introduction}

The gastrointestinal tract is commonly accompanied by non-Hodgkin lymphoma (NHL), and the stomach is the major site to be affected by $60 \%$ NHL patients with digestive tract involvement. ${ }^{1}$ Among lymphomas of the stomach, diffuse large B-cell lymphoma (DLBCL) and mucosa-associated lymphoid tissue lymphoma (MALT) are the two major types. ${ }^{2}$ Chemotherapy is recommended as the first-line treatment for GDLBCL according to the NCCN Guidelines and the Japanese gastric cancer treatment guidelines 2010 (ver. 3). Surgery is recommended as an urgent and palliative treatment for patients presenting with obstruction, bleeding, or severe perforation. ${ }^{3}$ The 
therapy of R-CHOP has obviously improved the therapeutic effects of GDLBCL patients, though the number of benefits patients is limited. Rituximab plus first-line chemotherapy treatment of GDLBCL reduces the incidence of central nervous system relapses. ${ }^{4}$ Due to the significant clinical and biological heterogeneity of GDLBCL patients, more efficient models or prognostic factors are needed to classify patients with various survival outcomes. Targeted therapies such as antibodies against programmed cell death 1 (PD-1) and its ligand (PD-L1) have a great prospect in the treatment of different malignant tumors. ${ }^{5}$ Recently, many clinical retrospective studies have exhibited responses to PD-1 antibodies for patients whose PD-L1 is expressed in tumor cells or tumor-infiltrating immune cells. ${ }^{6}$

MicroRNAs (miRNAs) are a group of noncoding RNAs with about 22 nucleotides, which can downregulate the target genes' expression through changing translational efficiency and stability of the target mRNAs. ${ }^{7}$ Currently, in the human genome, there are 1400 human miRNAs identified nearly, and each miRNA may influence many target genes. MiRNAs are expressed in a tissue-specific manner and produce an effect on cell differentiation, apoptosis, and proliferation. Recent trials have hinted that the abnormal expression of miRNAs is related to the occurrence of cancer. The members of the miR-34 family are regulated transcriptionally by $\mathrm{p} 53$, which are downregulated in acute myeloid leukemia and chronic lymphocytic leukemia., MiR-34a acts as a tumor suppressor by promoting apoptosis in some tumors. ${ }^{10}$ Interestingly, the upregulated PDL1 level is tightly linked to the downregulated miR-34a level in lung cancer and B-cell lymphomas, ${ }^{11,12}$ and PD$\mathrm{L} 1$ has been hinted to be a regulatory target of miR-34a. ${ }^{13}$ In this research, we demonstrated that PD-L1 was upregulated and miR-34a was downregulated in GDLBCL tissues. Through this research, we aimed to determine the prognostic implications of PD-L1 and miR-34a levels for clinical GDLBCL.

\section{Patients and Methods}

\section{Clinical Specimens}

From January 2010 to December 2018, a total of 109 patients that pathologically diagnosed with primary GDLBCL at Hunan Cancer Hospital were recruited for this study. Specimens used for qRT-PCR were preserved in liquid nitrogen. Samples used for IHC were fixed with formalin and embedded into paraffin. The diagnosis was established according to the histopathological and immunohistochemical criteria in the 2016 WHO classification system. The R-CHOP was administered every three weeks: day 1, rituximab $\left(375 \mathrm{mg} / \mathrm{m}^{2}\right)$; day 2 , vincristine $\left(1.4 \mathrm{mg} / \mathrm{m}^{2}\right.$, maximal dose $2 \mathrm{mg}$ ), doxorubicin ( $\left.50 \mathrm{mg} / \mathrm{m}^{2}\right)$, and cyclophosphamide $\left(750 \mathrm{mg} / \mathrm{m}^{2}\right)$; day 2 to 6 , prednisone (100 mg/day). It was administered for three to eight cycles. After R-CHOP was done, interventional field radiotherapy (IFRT) was supplied for extranodal disease, residual disease, and/or previous mass disease. The number of chemotherapy cycles and the adjustments of regimen dose were determined by physicians. The International Working Group Recommendations for Response Criteria with non-Hodgkin's lymphoma was used to assess treatment response. ${ }^{14}$ Retrospective research was made according to each patient's medical records.

\section{qRT-PCR}

The TRIzol reagent (Invitrogen, Carlsbad, CA, USA) was used to extract total RNA in tissue samples according to the manufacturer's instruction. qRT-PCR for PD-L1 and miR34a was performed using the ABI PRISM 7700 instrument (Perkin-Elmer Applied Biosystems, Foster City, CA, USA) with gene-specific primers and the SYBR Green (Takara, Tokyo, Japan). In brief, $1 \mu \mathrm{g}$ total RNA was reversetranscribed into cDNA in a total volume of $20 \mu \mathrm{L}$, and 1 $\mu \mathrm{L}$ cDNA was used as a template for qRT-PCR. Specific primers used for qRT-PCR are listed in Table 1. GAPDH and U6 were set as the internal control of mRNA and miRNA, respectively. ${ }^{15}$ The relative level of PD-L1 and miR-34a was calculated by the $2^{-\Delta \Delta \mathrm{Ct}}$ method. All experiments were independently repeated three times. The effect of miR-34a level in cancer tissues on prognosis was determined by Kaplan-Meier analysis according to the median.

Table I Primer Pairs Used for qRT-PCR

\begin{tabular}{|l|l|}
\hline Genes & Primer Sequences \\
\hline GAPDH & $\begin{array}{l}\text { FP: 5'-GCACCGTCAAGGCTGAGAAC-3' } \\
\text { RP: 5'-TGGTGAAGACGCCAGTGGA-3' }\end{array}$ \\
\hline PD-LI & $\begin{array}{l}\text { FP: 5'-AGATCAAAGAGAGCCTGCGG-3' } \\
\text { RP: 5'-AGGGGTCCTCCTTCAGGG-3' }\end{array}$ \\
\hline RNU6 & $\begin{array}{l}\text { FP: 5'-GCGCGTCGTGAAGCGTTC-3' } \\
\text { RP: 5'-GTGCAGGGTCCGAGGT-3' }\end{array}$ \\
\hline miR-34a & $\begin{array}{l}\text { FP: 5'-CGGTATCATTTGGCAGTGTCT-3' } \\
\text { RP: 5'-GTGCAGGGTCCGAGGT-3' }\end{array}$ \\
\hline
\end{tabular}

Abbreviations: GAPDH, glyceraldehyde-3-phosphate dehydrogenase; PD-LI, programmed death ligand-I; RNU6, U6 snRNA; miR-34a, microRNA-34a; FP, forward primer; RP, reverse primer. 


\section{Immunohistochemistry (IHC)}

The 5- $\mu \mathrm{m}$ paraffin-embedded tissue section was dewaxed in xylene and rehydrated in graded alcohols. Antigens were retrieved in boiled $1 \mu \mathrm{M}$ sodium citrate solution $(\mathrm{pH}=6.0)$ for two minutes. After blockade with normal goat serum for one hour, slices were sequentially incubated with the primary antibody anti-PD-L1 (PD-L1 antibody purchased from Proteintech) overnight at $4^{\circ} \mathrm{C}$ and the secondary antibody at room temperature for one hour, stained with $\mathrm{DAB}$ solution and counterstained with hematoxylin. Tissue slices were viewed at $400 \times$ magnification under inverted microscopy, and represented images were represented in figures. Three fields per section were analyzed.

We defined the criterion of PD-L1 positivity that tumor cells were positively stained by more than $5 \%$. Two observers scored $20 \%$ cases to assess reproducibility. Cases were supposed to be evaluable that more than a quarter of tissue was available for morphologic analysis and more than one positively staining tumor-infiltrating macrophage as a positive internal control.

Immunostaining for CD5, CD10, CD20, Bcl-2 and Bcl6 with antigen retrieval and antibody dilutions on paraffin sections was performed according to manufacturers' recommendations and previous report elsewhere. ${ }^{16}$

\section{Follow-Up}

The information of follow-up was gained from the patients or patients' relatives and information systems of our hospital that contained progression-free survival (PFS), overall survival (OS), and overall response rate (ORR). PFS was defined from the date of the pathological diagnosis to progression. OS was defined from the date of pathological diagnosis to death or the last follow-up. The response to first-line therapy consisted of complete remission (CR), partial response (PR), stable disease (SD), or progressive disease (PD). ORR was defined as CR plus PR. The patient's prognosis was analyzed.

\section{Statistical Analysis}

The normality of data distribution was tested by the Kolmogorov-Smirnov test. Pearson's chi-square test, independent-samples $t$-test, paired-samples $t$-test, Pearson's correlation, nonparametric Mann-Whitney $U$ or Wilcoxon signed ranks test, Spearman's rank correlation and logistic regression were used when appropriate. A univariate test and multivariate test were used to look for the influence of each clinical variable on prognosis. Data analysis was performed using SPSS version 22.0 (SPSS, Inc., Chicago, IL, USA). All data were mean $\pm \mathrm{SD}$ or median (range). ${ }^{*} P<0.05,{ }^{*} P<0.01$, $* * * P<0.001$ were considered statistically significant.

\section{Results \\ Clinicopathological Characteristics of GDLBCL}

Of the 109 patients in this study, the primary clinicopathologic characteristics were shown in Table 2. The median age was 53.7 years old (range $18-76$ years), and $62.4 \%$ of patients were less than 60 years. Few patients $(9.2 \%)$ had an unfavorable performance status (PS) (PS $\geq 2) .67$ patients were at Lugano stage I or II2, while the other 42 patients were at stage IIE or IV. Next, there were 4 patients (3.7\%) in a high-risk group, 22 patients $(20.2 \%)$ in a highintermediate risk group, 23 patients $(21.1 \%)$ in a lowintermediate risk group, and 60 patients $(55.1 \%)$ in the low-risk group, according to International Prognostic Index (IPI) scores. What's more, 49 patients (45\%) were Bcl-2 positive, while 60 patients $(55.1 \%)$ were $\mathrm{Bcl}-2$ negative.

\section{PD-LI Was Highly Expressed and miR-34a Was Lowly Expressed in GDLBCL}

The expression level of PD-L1 mRNA and miR-34a was determined by qRT-PCR in 109 tumor specimens with GDLBCL. According to the median of miR-34a expression level, patients were divided into low-level and high-level groups. MiR-34a was lowly expressed in 54 specimens (49.5\%), and significant difference (0.391 vs. 0.842, $P<0.001)$ was uncovered between the low-level and highlevel groups (Figure 1A, the right part). Moreover, a significantly negative correlation (Pearson's correlation, $r=$ -0.524, $P<0.001)$ existed between PD-L1 and miR-34a level (Figure 1B). Among 109 GDLBCL cases, 15 patients were surgically treated. PD-L1 mRNA level in cancer tissues of those 15 specimens was higher than that in paired noncancerous gastric tissues (1.694 vs. 1.000, $P<0.001$, Figure 1C), while the miR-34a level was just reversed ( 0.619 vs. $1.000, P<0.001$, Figure 1D).

We also examined the PD-L1 protein level by IHC in 109 FFPE specimens with GDLBCL. We defined the criterion of PD-L1 positivity that tumor cells were positively stained more than $5 \%$ (Figure $1 \mathrm{E}$ and $\mathrm{F}$ ). As a result, $\mathrm{PD}$ L1 was highly expressed in 66 specimens $(60.6 \%)$, and the relative level of PD-L1 mRNA in the IHC-positive group 
Table 2 Clinical Features of 109 Patients with GDLBCL According to PD-LI and miR-34a Expression

\begin{tabular}{|c|c|c|c|c|c|c|c|c|}
\hline \multirow[t]{2}{*}{ Groups } & \multirow[t]{2}{*}{ Clusters } & \multirow[t]{2}{*}{ N (\%) } & \multicolumn{3}{|c|}{ PD-LI } & \multicolumn{3}{|c|}{ miR-34a } \\
\hline & & & - & + & $P$ & - & + & $P$ \\
\hline \multirow[t]{2}{*}{ Age } & $\leq 60$ & 68 (62.4\%) & 28 & 40 & & 33 & 35 & \\
\hline & $>60$ & 41 (37.6\%) & 15 & 26 & & 21 & 20 & \\
\hline \multirow[t]{2}{*}{ Gender } & Male & 52 (47.7\%) & 21 & 31 & & 26 & 26 & \\
\hline & Female & 57 (52.3\%) & 22 & 35 & & 28 & 29 & \\
\hline \multirow[t]{2}{*}{ BMI } & $\leq 25$ & 88 (81.7\%) & 35 & 53 & & 42 & 46 & \\
\hline & $>25$ & 21 (18.3\%) & 8 & 13 & & 12 & 9 & \\
\hline \multirow[t]{2}{*}{$\mathrm{Hs}-\mathrm{CRP}$} & $\leq 6 \mathrm{mg} / \mathrm{l}$ & 90 (82.6\%) & 36 & 54 & & 45 & 45 & \\
\hline & $>6 \mathrm{mg} / \mathrm{l}$ & 19 (17.4\%) & 7 & 12 & & 9 & 10 & \\
\hline \multirow[t]{2}{*}{ ECOG PS } & $0-1$ & 99 (90.8\%) & 40 & 59 & & 48 & 51 & \\
\hline & $\geq 2$ & $10(9.2 \%)$ & 3 & 7 & & 6 & 4 & \\
\hline \multirow[t]{2}{*}{ Lugano stage } & $1-112$ & 67 (61.5\%) & 37 & 30 & $* * *$ & 26 & 41 & $* *$ \\
\hline & IIE-IV & 42 (38.5\%) & 6 & 36 & & 28 & 14 & \\
\hline \multirow[t]{2}{*}{ Serumal LDH } & Normal & 57 (52.3\%) & 33 & 24 & $* * *$ & 23 & 34 & $*$ \\
\hline & Elevated & 52 (47.7\%) & 10 & 42 & & 31 & 21 & \\
\hline \multirow{2}{*}{$\begin{array}{l}\text { Extranodal } \\
\text { site }\end{array}$} & $0-1$ & 91 (83.5\%) & 38 & 53 & & 42 & 49 & \\
\hline & $\geq 2$ & 18 (16.5\%) & 5 & 13 & & 12 & 6 & \\
\hline \multirow[t]{2}{*}{ B symptoms } & No & 57 (52.3\%) & 34 & 23 & $* * *$ & 18 & 39 & $* * *$ \\
\hline & Yes & 52 (47.7\%) & 9 & 43 & & 36 & 16 & \\
\hline \multirow[t]{2}{*}{ IPI } & $0-1$ & 60 (55.0\%) & 34 & 26 & $* * *$ & 23 & 37 & $*$ \\
\hline & $\geq 2$ & 49 (45.0\%) & 9 & 40 & & 31 & 18 & \\
\hline \multirow[t]{3}{*}{ R-IPI } & 0 & 21 (19.3\%) & 18 & 3 & $* *$ & 4 & 17 & $* *$ \\
\hline & $1-2$ & $62(56.9 \%)$ & 20 & 42 & & 32 & 30 & \\
\hline & $3-5$ & 26 (23.9\%) & 5 & 21 & & 18 & 8 & \\
\hline \multirow[t]{3}{*}{ Pathology } & GCB & 45 (4I.3\%) & 29 & 16 & $* * *$ & 13 & 32 & $* * *$ \\
\hline & Non- & $64(58.7 \%)$ & 14 & 50 & & 41 & 23 & \\
\hline & GCB & & & & & & & \\
\hline \multirow[t]{2}{*}{ CD5 } & - & $99(90.8 \%)$ & 39 & 60 & & 51 & 48 & \\
\hline & + & 10 (9.2\%) & 4 & 6 & & 3 & 7 & \\
\hline \multirow[t]{2}{*}{ CDIO } & - & 68 (62.4\%) & 30 & 38 & & 29 & 39 & \\
\hline & + & $4 \mid(37.6 \%)$ & 13 & 28 & & 25 & 16 & \\
\hline \multirow[t]{2}{*}{$\mathrm{Bcl}-2$} & - & $60(55.0 \%)$ & 18 & 42 & $*$ & 39 & 21 & $* * *$ \\
\hline & + & 49 (45.0\%) & 25 & 24 & & 15 & 34 & \\
\hline \multirow[t]{2}{*}{$\mathrm{Bcl}-6$} & - & $40(36.7 \%)$ & 19 & 21 & & 18 & 22 & \\
\hline & + & 69 (63.3\%) & 24 & 45 & & 36 & 33 & \\
\hline
\end{tabular}

Notes: -: Negative; +: Positive. Statistically significant, ${ }^{*} \mathrm{p}<0.05,{ }^{*} \mathrm{p}<0.01$, $* * * \mathrm{P}<0.001$.

Abbreviations: GDLBCL, gastric diffuse large B-cell lymphoma; PD-LI, programmed death ligand-I; miR-34a, microRNA-34a; BMI, body mass index; HsCRP, high-sensitivity C-reactive protein; ECOG PS, Eastern Cooperative Oncology Group Performance Status; LDH, lactate dehydrogenase; IPI, International Prognostic Index; R-IPI, revised International Prognostic Index; GCB, germinal center B-cell.

was significantly higher than that in the negative group (2.147 vs. 1.001, $P<0.001$ ) (Figure $1 \mathrm{~A}$, the left part). And significant Spearman's rank correlation was observed between PD-L1 protein level determined by IHC and
mRNA level examined by qRT-PCR $(\rho=0.712, P<0.001$, Table 3).

\section{Correlations Between the Expression of PD-LI, miR-34a, and Clinicopathological Features}

To characterize the clinical role of PD-L1 and miR-34a in GDLBCL furtherly, we tried to precisely identify the correlations of PD-L1 and miR-34a level with clinicopathological indicators, such as patient gender, age, BMI, Hs-CRP, ECOG PS, Lugano stage, serumal LDH, extranodal site tumor number, B symptoms, IPI, R-IPI, pathology subtype and expression level of CD5, CD10, Bcl-2, and Bcl-6. And no significant correlations between the expression level of PD-L1, miR-34a, and the main clinical features such as patient gender, age, BMI, Hs-CRP, ECOG PS, extranodal site tumor number, CD5, CD10 and Bcl-6 (Table 2). However, high level of PDL1 and low level of miR-34a exhibited a high incidence with advanced Lugano stage (Stage IIE and IV, $P<0.001$ and $P<0.01$, respectively), elevated serumal LDH levels $(P<0.001$ and $P<0.05$, respectively), B symptoms present ( $P<0.001$ and $P<0.001$, respectively), non-GCB subtype $(P<0.001$ and $P<0.001$, respectively) and negative Bcl-2 expression $(P<0.05$ and $P<0.001$, respectively). Furthermore, PD-L1 high-level and miR-34a low-level groups had more patients with IPI scores of 2 or greater $(P<0.001$ and $P<0.05$, respectively) and poor R-IPI $(P<0.01$ and $P<0.01$, respectively).

\section{Relationships Between Treatment Outcomes and Expression Level of PD-LI}

\section{and miR-34a}

Table 4 showed the response details to first-line treatment (R-CHOP \pm IFRT). Following initial therapy, the ORR was $82.6 \%$, and CR was achieved in 72 patients $(66.1 \%)$, but 27 patients (24.8\%) showed disease progression or relapses subsequently. Notably, PD-L1 and miR-34a levels were significantly correlated with both CR efficacy and progression/relapse rate. The PD-L1-positive group had a lower CR rate and a higher progression/relapse rate than the negative group (CR rate, $32.1 \%$ vs. $33.9 \%$, $P=0.005$; progression/relapse rate, $20.2 \%$ vs. $4.6 \%$, $P=0.010$; Table 4). Moreover, the miR-34a-positive group had a higher CR rate and a lower progression/ relapse rate than the miR-34a-negative group (CR rate, $40.4 \%$ vs. $25.7 \%, P=0.015$; relapse/progression rate, $6.4 \%$ vs. $18.3 \%, P=0.003$; Table 4$)$. In order to exclude 

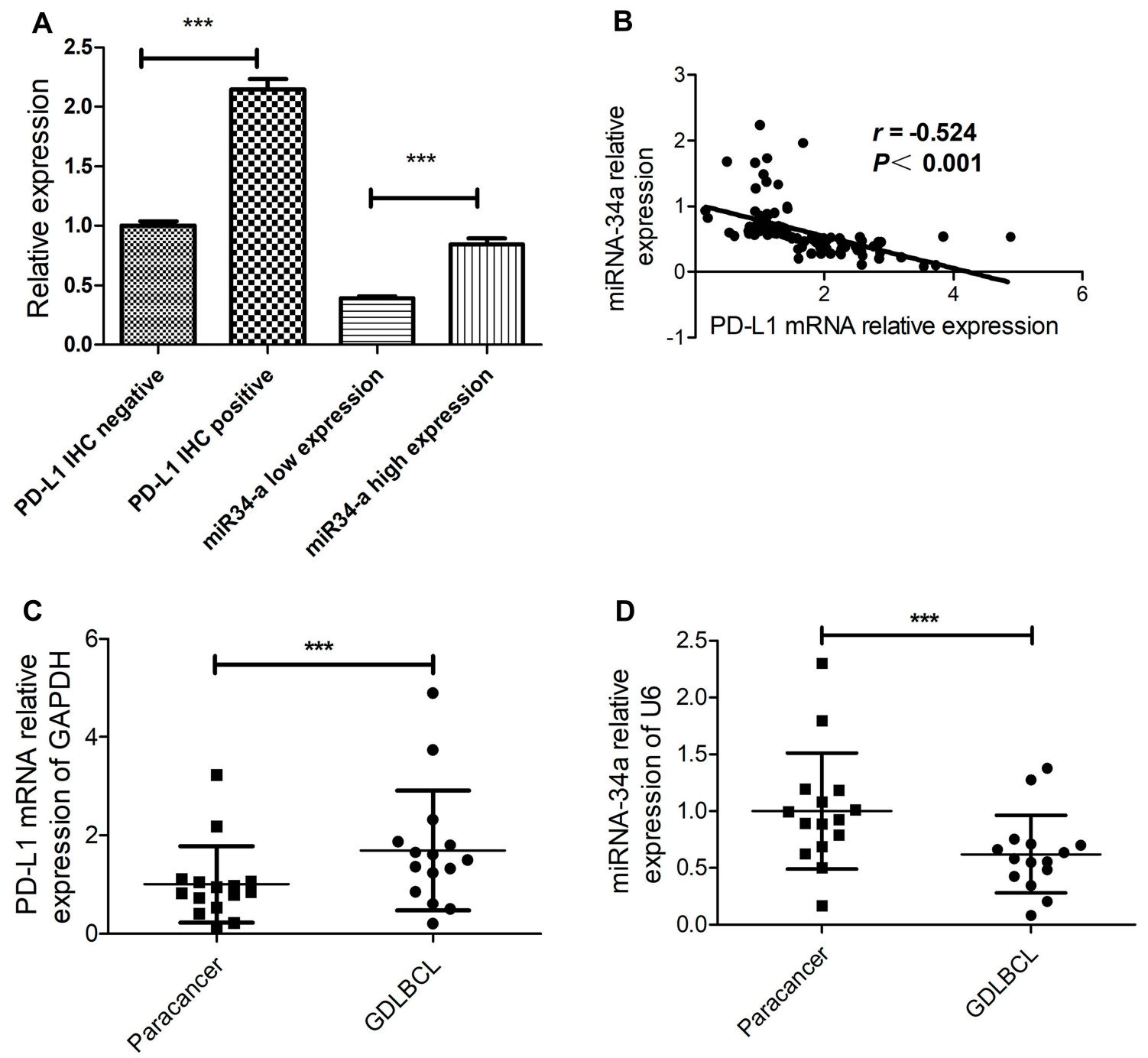

E
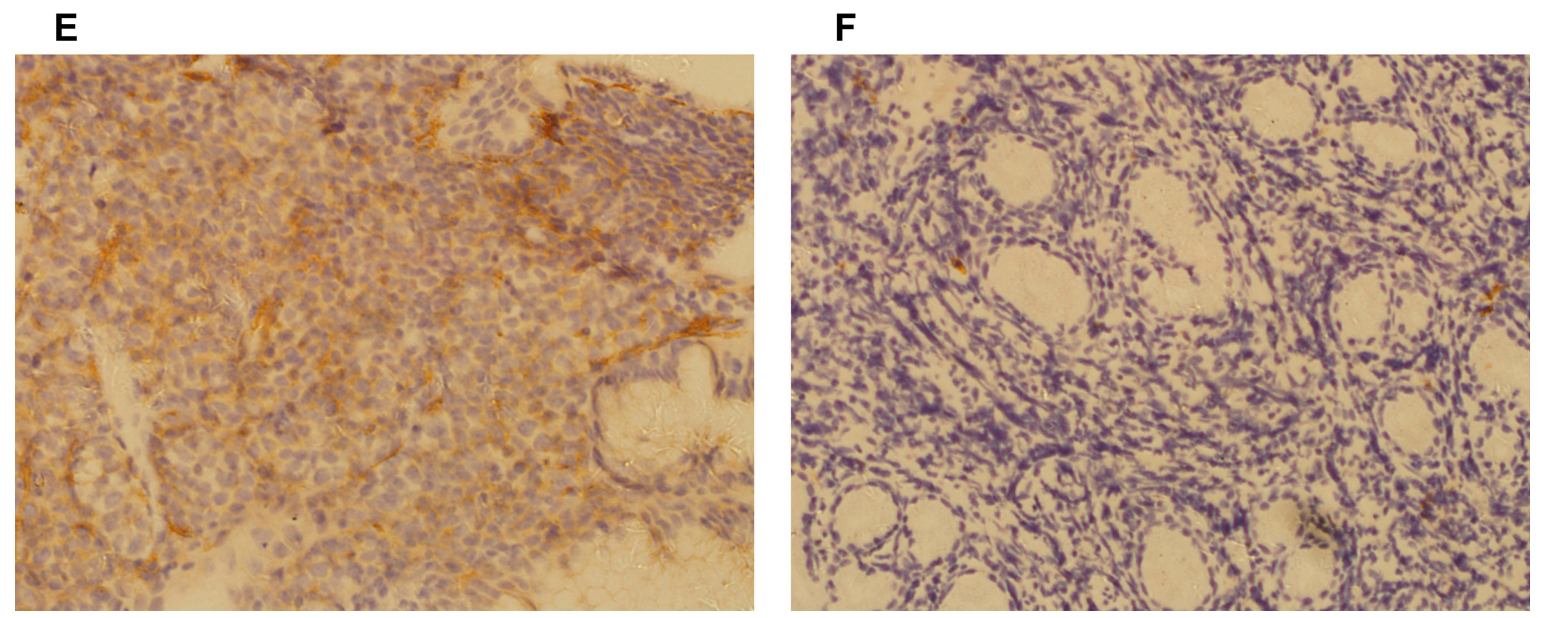

Figure I PD-LI is overexpressed and miR-34a is low expressed in GDLBCL tissues. (A) the left part, the relative expressions of PD-LI mRNA in the IHC-positive group were higher than in IHC-negative group; the right part, the relative expressions of miR-34a mRNA in the high expression group were higher than in low expression group. GAPDH and $U 6$ were set as the internal control of mRNA and miRNA, respectively. Statistically significant, $* * * P<0.001$. (B) Pearson's correlation analysis showed that there was significant correlation between relative expression of PD-LI and miR-34a expression. GAPDH and U6 were set as the internal control of mRNA and miRNA, respectively. (C) PD-LI mRNA expression was obviously higher than that in adjacent noncancerous gastric tissues. GAPDH was set as the internal control of mRNA. Statistically significant, ${ }^{*} * P<0.001$. (D) miR-34a expression was obviously lower than that in adjacent noncancerous gastric tissues. U6 was set as the internal control of miRNA. Statistically significant, $* * * P<0.001$. (E) PD-LI is highly expressed in GDLBCL tissues $(\times 400)$. (F) PD-LI was weakly expressed in GDLBCL tissues ( $\times 400)$. Abbreviations: IHC, immunohistochemistry; PD-LI, programmed death ligand-I; miR-34a, microRNA-34a; GAPDH, glyceraldehyde-3-phosphate dehydrogenase; RNU6, U6 snRNA; GDLBCL, primary gastric diffuse large B-cell lymphoma. 
Table 3 Relationship Between PD-LI Protein Level and mRNA Level

\begin{tabular}{|l|l|l|l|l|}
\hline Groups & No. of Patients & PD-LI mRNA & $\rho \#$ & $P$ \\
\hline PD-LI IHC negative & 44 & $1.001 \pm 0.2610$ & 0.712 & $<0.001$ \\
PD-LI IHC positive & 66 & $2.147 \pm 0.685$ & & \\
\hline
\end{tabular}

Note: \#Spearman's rank correlation.

Abbreviations: PD-LI, programmed death ligand-I; IHC, immunohistochemistry.

Table 4 Comparison of Treatment Outcomes Based on PD-LI and miR-34a Expression Level

\begin{tabular}{|l|l|l|l|l|l|l|l|}
\hline \multirow{2}{*}{ Features } & \multirow{2}{*}{ N (\%) } & \multicolumn{2}{|l|}{ PD-LI } & \multicolumn{3}{|l|}{ miR-34a } \\
\cline { 3 - 7 } & & - & + & $P$ & - & + & $P$ \\
\hline Response & & & & 0.005 & & & 0.015 \\
CR & $72(66.1 \%)$ & 37 & 35 & & 28 & 44 & \\
PR & $18(16.5 \%)$ & 3 & 15 & & 13 & 5 & \\
SD & $6(5.5 \%)$ & 1 & 5 & & 5 & 1 & \\
PD & $13(11.9 \%)$ & 2 & 11 & & 8 & 5 & \\
\hline Progression/relapse & & & & 0.010 & & & 0.003 \\
No & $82(75.2 \%)$ & 38 & 34 & & 34 & 48 & \\
Yes & $27(24.8 \%)$ & 5 & 22 & & 20 & 7 & \\
\hline Treatment modality & & & & 0.079 & & & 0.176 \\
R-CHOP & $94(86.2 \%)$ & 34 & 60 & & 49 & 45 & \\
R-CHOP+IFRT & $15(13.8 \%)$ & 9 & 6 & & 5 & 10 & \\
\hline
\end{tabular}

Notes: -: Negative; +: Positive.

Abbreviations: PD-LI, programmed death ligand-I; miR-34a, microRNA-34a; CR, complete response; PR, partial response; SD, stable disease; PD, progressive disease; R-CHOP, rituximab plus cyclophosphamide, doxorubicin, vincristine, and Prednisone; IFRT, involved-field radiotherapy.

possible treatment-related bias, we compared the treatment type with the expression of PD-L1 and miR-34a. Patients treated with R-CHOP alone and patients treated with R-CHOP+IFRT were applied for this comparison, whereas no significant difference between PD-L1- and miR-34anegative and -positive groups $(P=0.079$ and $P=0.176$, respectively; Table 4).

\section{Survival Analysis and Follow-Up}

During an average follow-up of 56 months (range: $2.7-87.8$ months), there were 38 deaths (34.9\%). Comparison of patient's survival outcomes between the negative and positive PD-L1 groups, the positive PD-L1 expression group showed inferior PFS and OS $(P=0.004$ and 0.001 , respectively; Figure 2A and B). Besides, the negative miR-34a expression group showed inferior PFS and OS when compared with the miR-34a-positive group ( $P=0.009$ and 0.009 , respectively; Figure $2 \mathrm{C}$ and D).

\section{Univariate Analysis and Multivariate Analysis of Prognostic Factors}

So as to identify the potential significant prognostic factors, a univariate analysis of each main factor was performed concerning the prognosis of GDLBCL patients. The hazard ratio and $P$ value of each factor were used to predict the difference in prognosis. Then, a multivariate Cox proportional hazards model was used to determine the significance of each factor. Through univariate analysis, factors were gradually included in the model, which determined that the significant prognostic factors in PFS and OS of GDLBCL patients were PD-L1, miR-34a, Lugano stage, presence of B symptoms, IPI and Bcl-2 (Table 5). The results of the multivariate analysis indicated that PFS could be predicted based on PD-L1, miR-34a, and IPI (Table 5), and that OS could be predicted based on PDL1 and miR-34a (Table 6).

\section{Discussion}

There are some breakthroughs in cancer diagnosis and chemotherapy. Recently, immunotherapies targeting PD1/PD-L1 have become effective strategies for the treatment of some types of tumors. ${ }^{5}$ In this research, PD-L1-negative expression and miR-34a-positive expression were favorable prognostic impacts for GDLBCL patients. Another notable finding of this research was that the low expression of PD-L1 was associated with the high expression of miR-34a. Recent research demonstrates that immune checkpoint PD-L1 is regulated by miR-34a in DLBCL. ${ }^{12}$ Hence, careful consideration of PD-L1 and miR-34a in GDLBCL may be important for selecting PD-1/PD-L1 checkpoint blockades.

There are limited trials on the predictive value of PDL1 in GDLBCL, and most of which are retrospective studies with a controversial conclusion. A lot of previous studies reveal that PD-L1 upregulation is linked to an unfavorable prognosis. However, some investigators find no prognostic significance based on PD-L1 expression, and the others demonstrate that PD-L1 is a beneficial prognostic in GDLBCL. ${ }^{17}$ In previous studies, the cut-off values of PD-L1 positivity are varied from $5 \%$ to $30 \%$, while the percentages of PD-L1-positive DLBCL are varied from $11 \%$ to $75 \% .{ }^{18}$ This heterogeneity shows that even experienced hematopathologists can hardly distinguish between PD-L1-positive cells and PD-L1-negative cells. Recently, Chen et $\mathrm{al}^{19}$ reported the prevalence of 
PD-L1-positive DLBCL, in which the use of PD-L1/Pax5 double staining in selected cases may reduce the heterogeneities. However, it is still subjective and difficult to promote worldwide. In our study, qRT-PCR was used to detect PD-L1 mRNA expression levels, and IHC was used to detect PD-L1 protein levels. The relative expression of PD-L1 mRNA in the IHC-negative group was lower than in IHC-positive group. There was a significant correlation (Spearman's rank correlation, $\rho=0.712, P<0.001$ ) between PD-L1 protein level and mRNA level. Thus, we might use the qRT-PCR method to get more accurate PD-L1 expression, instead of IHC.

Our study revealed a significant correlation between PD-L1-positive expression and non-GCB, which was similar to former research. ${ }^{18}$ Besides, we found that positive PD-L1 expression was linked to Bcl-2 expression significantly. And the increased PD-L1 level was usually related to poor clinical features, such as B symptoms and IPI scores of 2 or greater. PD-L1 expression induces worse OS irrespective of using rituximab. ${ }^{20}$ Therefore, GDLBCL patients with high PD-L1 expression do not benefit from first-line treatment. ${ }^{21}$ In literature reports, tumor cells that express PD-L1 have many mechanisms to escape T-cell immunity. ${ }^{22}$ PD-1/PD-L1 pathway induces apoptosis of $\mathrm{PD}-1^{+}$tumor-associated antigen-specific $\mathrm{T}$ cells, which is one of its important mechanisms. ${ }^{23}$ Another possible reason is that chemotherapeutic resistance may arise from positive PD-L1 expression partly, which may link to poor prognosis. ${ }^{21}$ These findings suggest that PD-L1 expression may be favorable to discover GDLBCL patients who have a high disease progression risk.

MiRNAs can affect lineage choice or critical developmental checkpoints in the hematopoietic process. ${ }^{24} \mathrm{MiR}-34 \mathrm{a}$ is one of the tumor suppressor miRNAs, which is the key
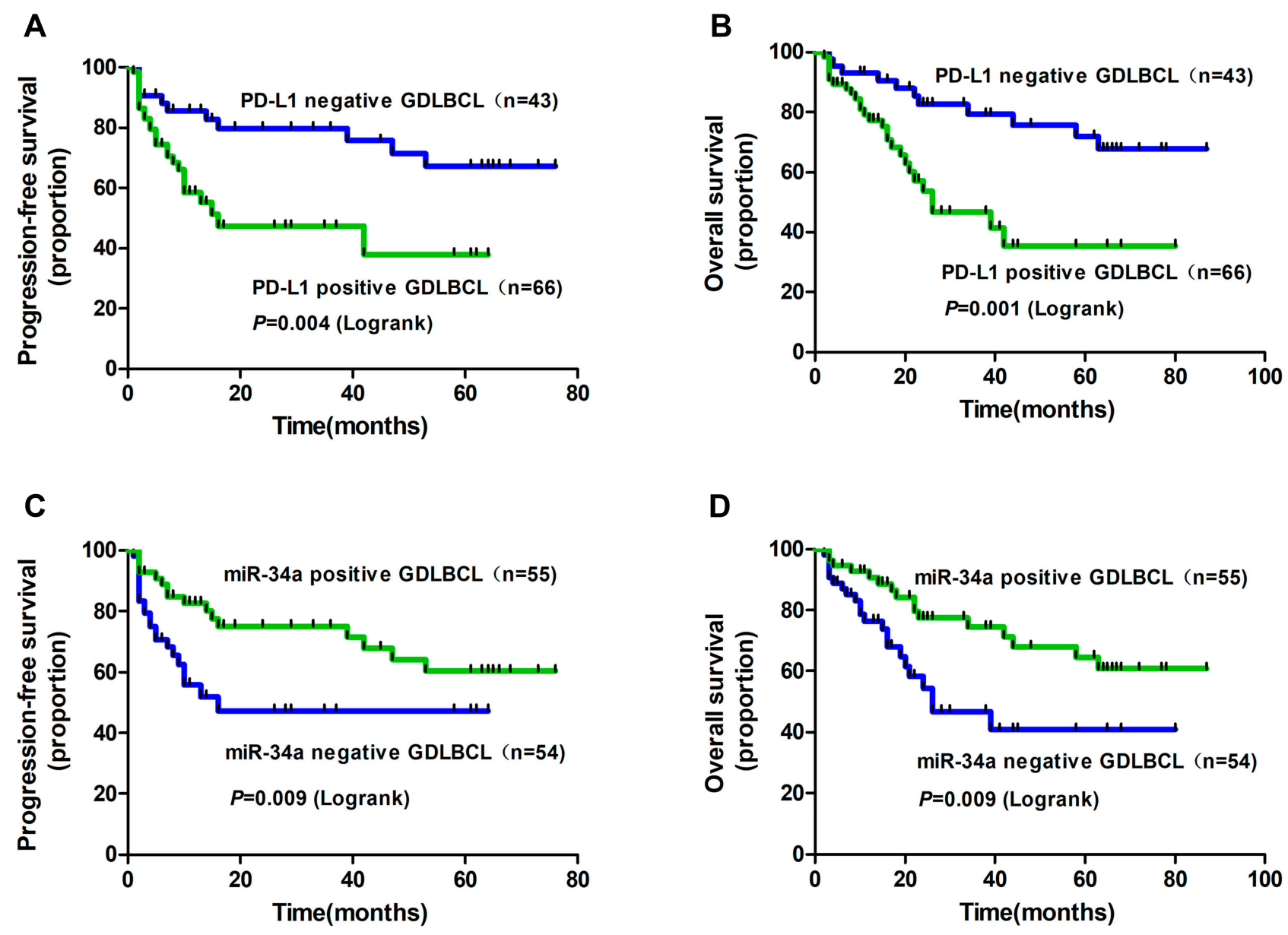

Figure 2 Comparison of survival outcome according to PD-LI and miR-34a expression. (A) Patients with positive PD-LI expression showed significant inferior PFS. (B) Patients with positive PD-LI expression showed significantly inferior OS. (C) Patients with negative miR-34a expression showed significant inferior PFS. (D) Patients with negative miR-34a expression showed significantly inferior OS.

Abbreviations: PD-LI, programmed death ligand-I; miR-34a, microRNA-34a; GDLBCL, primary gastric diffuse large B-cell lymphoma; PFS, progression-free survival; OS, overall survival. 
Table 5 Univariable Analysis of Clinicopathological Prognostic Factors for PFS and OS in GDLBCL Patients

\begin{tabular}{|c|c|c|c|c|c|c|}
\hline \multirow[t]{2}{*}{ Risk Factor } & \multicolumn{3}{|l|}{ PFS } & \multicolumn{3}{|l|}{ os } \\
\hline & HR & $95 \% \mathrm{Cl}$ & $\mathbf{P}$ & HR & $95 \% \mathrm{Cl}$ & $\mathbf{P}$ \\
\hline PD-LI & 2.841 & $1.368-5.901$ & 0.005 & 3.211 & $1.546-6.672$ & 0.002 \\
\hline miR-34a & 0.426 & $0.220-0.825$ & 0.011 & 0.422 & $0.218-0.818$ & 0.011 \\
\hline Age & 1.424 & $0.75 I-2.70 I$ & 0.280 & 1.552 & $0.818-2.943$ & 0.179 \\
\hline Gender & 1.125 & $0.593-2.134$ & 0.718 & 1.095 & $0.578-2.075$ & $0.78 I$ \\
\hline BMI & 1.762 & $0.828-3.751$ & 0.141 & 1.515 & $0.710-3.233$ & 0.283 \\
\hline $\mathrm{Hs}-\mathrm{CRP}$ & 0.825 & $0.345-1.974$ & 0.665 & 0.849 & $0.355-2.032$ & 0.849 \\
\hline ECOG PS & 1.907 & $0.743-4.893$ & 0.180 & 1.708 & $0.666-4.381$ & 0.265 \\
\hline Lugano stage & 2.043 & $1.067-3.913$ & 0.031 & 1.794 & $0.944-3.410$ & 0.074 \\
\hline Serumal LDH & 1.500 & $0.786-2.863$ & 0.218 & 1.533 & $0.805-2.917$ & 0.194 \\
\hline Extranodal site & 1.569 & $0.689-3.574$ & 0.284 & 1.087 & $0.478-2.47 \mid$ & 0.842 \\
\hline B symptoms & 2.195 & I. $138-4.232$ & 0.019 & 2.446 & $1.264-4.730$ & 0.008 \\
\hline IPI & 2.772 & I.428-5.378 & 0.003 & 2.556 & I.327-4.927 & 0.005 \\
\hline R-IPI & 1.567 & $0.970-2.533$ & 0.067 & 1.430 & $0.905-2.260$ & 0.125 \\
\hline Pathology & 1.954 & $0.980-3.896$ & 0.057 & 1.916 & $0.962-3.819$ & 0.064 \\
\hline CD5 & 1.636 & $0.682-3.920$ & 0.270 & 1.709 & $0.713-4.094$ & 0.229 \\
\hline CDIO & 1.003 & $0.512-1.965$ & 0.993 & 0.947 & $0.484-1.852$ & 0.873 \\
\hline $\mathrm{Bcl}-2$ & 0.504 & $0.257-0.987$ & 0.046 & 0.549 & $0.279-1.079$ & 0.082 \\
\hline $\mathrm{Bcl}-6$ & 1.483 & $0.746-2.947$ & 0.261 & 1.512 & $0.762-3.001$ & 0.237 \\
\hline
\end{tabular}

Abbreviations: HR, hazard ratio; Cl, confidence interval; PFS, progression-free survival; OS, overall survival; PD-LI, programmed death ligand-I; miR-34a, microRNA-34a; BMI, body mass index; Hs-CRP, high-sensitivity C-reactive protein; ECOG PS, Eastern Cooperative Oncology Group Performance Status; LDH, lactate dehydrogenase; IPI, International Prognostic Index; R-IPI revised International Prognostic Index.

Table 6 Multivariate Analysis of Clinicopathological Prognostic Factors for PFS and OS in GDLBCL Patients

\begin{tabular}{|l|l|l|l|l|l|l|}
\hline \multirow{2}{*}{ Risk Factor } & \multicolumn{2}{l}{ PFS } & \multicolumn{2}{l|}{ OS } & \multicolumn{3}{l|}{} \\
\cline { 2 - 7 } & HR & $\mathbf{9 5 \%} \mathbf{C l}$ & $\mathbf{P}$ & HR & $\mathbf{9 5 \%} \mathbf{C I}$ \\
\hline PD-LI & 2.277 & $1.030-5.034$ & 0.042 & 1.360 & $1.169-5.583$ & 0.029 \\
miR-34a & 0.502 & $0.280-0.961$ & 0.045 & 0.506 & $0.285-0.957$ & 0.048 \\
B symptoms & 1.078 & $0.484-2.403$ & 0.855 & 1.487 & $0.679-3.259$ & 0.321 \\
IPI & 2.234 & $1.008-4.591$ & 0.048 & 1.872 & $0.866-4.047$ & 0.111 \\
\hline
\end{tabular}

Abbreviations: HR, hazard ratio; Cl, confidence interval; PFS, progression-free survival; OS, overall survival; PD-LI, programmed death ligand-I; miR-34a, microRNA34a; IPI, International Prognostic Index.

regulatory factor of a tumor suppressor. It is downregulated in some kinds of tumors and participates in the occurrence and development of tumors. ${ }^{10}$ It is reported that the constitutive expression of miR-34a blocked the development of $\mathrm{B}$ cells in the transitional period from pre-B cells to pre-B cells, resulting in a decrease of mature B cells. ${ }^{10}$ As a direct activator of p53, miR-34a is involved in the p53 network. Wild type p53 lead miR-34a transcription, and miR-34a targets a variety of molecules involved in cell transformation and carcinogenesis. ${ }^{10,24}$ Usually, miR-34a is often downregulated in some kinds of tumors. ${ }^{12}$ In our research, we found that the expression of miR-34a was decreased in GDLBCL than that in adjacent noncancerous gastric tissues, aligning with previous viewpoints. ${ }^{15}$ Our results also found that low expression of miR-34a was linked to non-GCB subtype and $\mathrm{Bcl}-2$, and was usually related to poor clinical features, such as B symptoms and IPI scores of 2 or greater. Previous research shows that the expression of miR-34a is downregulated in GDLBCL and may be related to the patient's progression and metastasis by targeting Bcl-2, which has been confirmed as a regulatory target of miR-34a. ${ }^{15}$ Moreover, the miR-34a low expression is linked to the worse outcome of GDLBCL. The expression level of miR-34a is reduced in a non-GCB type of DLBCL cells and tumor tissues. The overall survival rate of patients with lower miR-34a is worse, while increased expression of miR-34a makes nonGCB DLBCL cells respond to doxorubicin treatment. ${ }^{25}$ These results suggested that miR-34a was an important 
tumor suppressor and a potential biomarker for treatment and prognostic of GDLBCL.

Recent research reveals that miR-34a regulates immune checkpoint PD-L1 in lung cancer and B-cell lymphomas. ${ }^{11,12}$ In our research, we found that the low level of PD-L1 was significantly linked to a high level of miR-34a. And patients with PD-L1 high expression and miR-34a low expression had a high incidence of advanced Lugano stage (IIE or IV), elevated serumal LDH levels, B symptoms present, nonGCB subtype, and negative Bcl-2 expression. What's more, the PD-L1 high expression and miR-34a low expression groups were linked to IPI scores of 2 or greater and poor R-IPI. Whereas the PD-L1-negative expression and miR34a-positive expression were favorable prognostic factors in GDLBCL. What are the reasons or mechanisms that such down-regulation or up-regulation of these biomarkers influenced prognosis? The following reasons may relate to this question. Firstly, miR-34a functions as a tumor suppressor gene and links the p53 network by FOXP1 and Bcl-2. ${ }^{15}$ Additionally, PD-L1 expression is regulated by $\mathrm{p} 53$ via miR$34 \mathrm{a} .{ }^{12}$ In keeping with these, PD-L1 and miR-34a were uncovered to be independent prognostic factors for PFS and OS in GDLBCL patients treated with R-CHOP in our study.

In summary, our results show that approximately one-half of GDLBCL patients are positive for PD-L1 and negative for miR-34a. Positive PD-L1 expression and negative miR-34a expression are linked to a high prevalence of elevated IPI scores and elevated LDH levels. Positive PD-L1 expression and negative miR-34a expression are also linked to high progression/relapse rates and low $\mathrm{CR}$ rates after the firstline treatment of R-CHOP. PD-L1 and miR-34a are independent prognostic factors for PFS and OS in GDLBCL patients treated with R-CHOP. While it needs more trials to confirm our findings and to better understand the biological functions of PD-L1 and miR-34a in GDLBCL. The PD-L1-positive and miR-34a-negative patients are not rare in GDLBCL but have a worse clinical prognosis. In contrast, low PD-L1 and high miR-34a expression are identified to be a favorable prognostic group, though their prognostic impact could be different in some GDLBCL. This problem should be further settled in future research to choose superior candidates as PD-1/PD-L1 checkpoint blockades.

\section{Consent to Participate and Ethics Approval}

All tissue specimens used in this study were obtained from Hunan Cancer Hospital with informed consent from patients.
All procedures followed in experiments were following the ethical commission of Hunan Cancer Hospital and were complied with the Declaration of Helsinki.

\section{Acknowledgments}

This study was supported by Industry Research Project from the Development and Reform Commission of Hunan Province (2015-99, to Chaohui Zuo), and Key Research Project from the Hunan Provincial Science and Technology Department (2017k2130, to Chaohui Zuo). We thank Chen Liu for assistance with the writing of the manuscript.

\section{Disclosure}

Chaohui Zuo is currently investigating the clinical treatment and molecular biology of the recurrence and metastasis for digestive oncology as a researcher. Yajun $\mathrm{Li}$ is currently investigating the clinical treatment and molecular biology of the recurrence and metastasis for digestive lymphoma as a researcher. The authors report no other conflicts of interest in this work.

\section{References}

1. Sabattini E, Bacci F, Sagramoso C, Pileri SA. WHO classification of tumours of haematopoietic and lymphoid tissues in 2008: an overview. Pathologica. 2010;102(3):83-87.

2. Howell JM, Auer-Grzesiak I, Zhang J, Andrews CN, Stewart D, Urbanski SJ. Increasing incidence rates, distribution and histological characteristics of primary gastrointestinal non-hodgkin lymphoma in a North American population. Can J Gastroenterol. 2012;26 (7):452-456. doi:10.1155/2012/480160

3. Zelenetz AD, Gordon LI, Wierda WG, et al. Diffuse large B-cell lymphoma version 1.2016. J Natl Compr Canc Netw. 2016;14 (2):196-231. doi:10.6004/jnccn.2016.0023

4. Sohn BS, Kim SM, Yoon DH, et al. The comparison between CHOP and R-CHOP in primary gastric diffuse large B cell lymphoma. Ann Hematol. 2012;91(11):1731-1739. doi:10.1007/s00277-012-1512-4

5. Brahmer JR, Tykodi SS, Chow LQ, et al. Safety and activity of anti-PD-L1 antibody in patients with advanced cancer. $N$ Engl $J$ Med. 2012;366(26):2455-2465. doi:10.1056/NEJMoa1200694

6. Herbst RS, Soria JC, Kowanetz M, et al. Predictive correlates of response to the anti-PD-L1 antibody MPDL3280A in cancer patients. Nature. 2014;515(7528):563-567. doi:10.1038/nature14011

7. Nana-Sinkam SP, Croce CM. Clinical applications for microRNAs in cancer. Clin Pharmacol Ther. 2013;93(1):98-104. doi:10.1038/ clpt.2012.192

8. Adams BD, Anastasiadou E, Esteller M, He L, Slack FJ. The inescapable influence of noncoding RNAs in cancer. Cancer Res. 2015;75(24):5206-5210. doi:10.1158/0008-5472.CAN-15-1989

9. Wang LQ, Kwong YL, Wong KF, et al. Epigenetic inactivation of mir-34b/c in addition to mir-34a and DAPK1 in chronic lymphocytic leukemia. J Transl Med. 2014;12:52. doi:10.1186/1479-5876-12-52

10. Hermeking H. The miR-34 family in cancer and apoptosis. Cell Death Differ. 2010;17(2):193-199. doi:10.1038/cdd.2009.56

11. Cortez MA, Ivan C, Valdecanas D, et al. PDL1 regulation by p53 via miR-34. J Natl Cancer Inst. 2016;108:1. doi:10.1093/jnci/djv303 
12. Anastasiadou E, Stroopinsky D, Alimperti S, et al. Epstein-barr virus-encoded EBNA2 alters immune checkpoint PD-L1 expression by downregulating miR-34a in B-cell lymphomas. Leukemia. 2019;33(1):132-147. doi:10.1038/s41375-018-0178-x

13. Wang X, Li J, Dong K, et al. Tumor suppressor miR-34a targets PD-L1 and functions as a potential immunotherapeutic target in acute myeloid leukemia. Cell Signal. 2015;27(3):443-452. doi:10.1016/j. cellsig.2014.12.003

14. Barnes JA, Redd R, Fisher DC, et al. Panobinostat in combination with rituximab in heavily pretreated diffuse large B-cell lymphoma: results of a Phase II study. Hematol Oncol. 2018;36(4):633-637. doi:10.1002/hon. 2515

15. He M, Gao L, Zhang S, et al. Prognostic significance of miR-34a and its target proteins of FOXP1, p53, and BCL2 in gastric MALT lymphoma and DLBCL. Gastric Cancer. 2014;17(3):431-441. doi:10.1007/s10120-013-0313-3

16. Malipatel R, Patil M, Pritilata Rout P, Correa M, Devarbhavi H. Primary gastric lymphoma: clinicopathological profile. Euroasian $J$ Hepatogastroenterol. 2018;8(1):6-10. doi:10.5005/jp-journals-100181250

17. Zhao S, Zhang M, Zhang Y, et al. The prognostic value of programmed cell death ligand 1 expression in non-hodgkin lymphoma: a meta-analysis. Cancer Biol Med. 2018;15(3):290-298. doi:10.20892/j. issn.2095-3941.2018.0047

18. Kiyasu J, Miyoshi H, Hirata A, et al. Expression of programmed cell death ligand 1 is associated with poor overall survival in patients with diffuse large B-cell lymphoma. Blood. 2015;126(19):2193-2201. doi:10.1182/blood-2015-02-629600
19. Chen BJ, Chapuy B, Ouyang J, et al. PD-L1 expression is characteristic of a subset of aggressive B-cell lymphomas and virus-associated malignancies. Clin Cancer Res. 2013;19(13):3462-3473. doi:10.1158/1078-0432.CCR-13-0855

20. Sun C, Jia Y, Wang W, et al. Integrative analysis of PD-L1 DNA status, mRNA status and protein status, and their clinicopathological correlation, in diffuse large B-cell lymphoma. Histopathology. 2019;74(4):618-628. doi:10.1111/his.13765

21. Liu J, Quan L, Zhang C, Liu A, Tong D, Wang J. Over-activated PD-1/PD-L1 axis facilitates the chemoresistance of diffuse large B-cell lymphoma cells to the CHOP regimen. Oncol Lett. 2018;15 (3):3321-3328. doi:10.3892/ol.2017.7682

22. Zou W, Chen L. Inhibitory B7-family molecules in the tumour microenvironment. Nat Rev Immunol. 2008;8(6):467-477. doi: $10.1038 /$ nri2326

23. Dong H, Strome SE, Salomao DR, et al. Tumor-associated B7-H1 promotes T-cell apoptosis: a potential mechanism of immune evasion. Nat Med. 2002;8(8):793-800. doi:10.1038/nm730

24. Chen CZ, Li L, Lodish HF, Bartel DP. MicroRNAs modulate hematopoietic lineage differentiation. Science. 2004;303(5654):83-86. doi:10.1126/science. 1091903

25. Marques SC, Ranjbar B, Laursen MB, et al. High miR-34a expression improves response to doxorubicin in diffuse large B-cell lymphoma. Exp Hematol. 2016;44(4):238-246.e232. doi:10.1016/j. exphem.2015.12.007

\section{Publish your work in this journal}

Cancer Management and Research is an international, peer-reviewed open access journal focusing on cancer research and the optimal use of preventative and integrated treatment interventions to achieve improved outcomes, enhanced survival and quality of life for the cancer patient.
The manuscript management system is completely online and includes a very quick and fair peer-review system, which is all easy to use. Visit http://www.dovepress.com/testimonials.php to read real quotes from published authors. 